\title{
Photon Gas Is the Medium of Electromagnetic Waves
}

\author{
Jikang Chen \\ College of Physics, Nanjing Normal University, Nanjing, China \\ Email:chenjikang@njnu.edu.cn
}

How to cite this paper: Chen, J.K. (2018) Photon Gas Is the Medium of Electromagnetic Waves. World Journal of Mechanics, 8, 26-45.

https://doi.org/10.4236/wjm.2018.82003

Received: January 1, 2018

Accepted: February 11, 2018

Published: February 14, 2018

Copyright (C) 2018 by author and Scientific Research Publishing Inc. This work is licensed under the Creative Commons Attribution International License (CC BY 4.0).

http://creativecommons.org/licenses/by/4.0/

\begin{abstract}
The aim of this paper is to find the carrier or medium of electromagnetic waves that has been searched for many years. The thermal radiation is composed of the shot noise and the wave noise, so the Planck formula can be separated mathematically into two parts. Assume every photon has the same proper magnetic moment and an equal rest mass which has been estimated from the cosmic background temperature in space where the photon gas is at an open state of thermal equilibrium. The magnetic-electric dynamic equation is established that means magnetic curl caused electric change, and the electric-magnetic dynamic equation is established that means electric curl caused magnetic change. After the wave equation is caught, it is clear that the photon gas is the medium of electromagnetic waves in vacuum.
\end{abstract}

\section{Keywords}

Photon’s Rest Mass, Photon's Proper Magnetic Moment, Photon Gas

\section{Introduction}

Many people have been searching for the carrier or medium of the Maxwell's electromagnetic waves since the last century, and some scientists thought that the photon gas may be the medium, but the dynamic equations hasn't been caught. After the experiment of Michelson and Morley, the searching job was abandoned in the face of the almost universal adherence of physicists to the purely interpretation that the electromagnetic wave is carried by the energy of itself. To prove that the photon gas is the carrier or medium of electromagnetic waves, the magnetic-electric and the electric-magnetic dynamic equations must be established. The origin ideas is based on Planck formula, Einstein Special Relativity, and E. M. Purcell's Electricity and Magnetism, all that is come from textbooks. When dealing with the theoretical derivation, it is necessary that 
some general physics concepts should be rechecked carefully:

1) Defined the force based on the acceleration which is more fundamental than momentum, according to the principle of Einstein's special relativity and between the visual system $S$ where the observer is rest and the proper system $S$, where the object is rest, the transformations of the acceleration, the inertial mass, the force and the torques (Figures S1-S3, Equation (S1)-(S5)) can be obtained.

2) The magnetic field $B$ should be defined based on the line current. There will be trouble if defined $B$ based on the magnetic moment which is a tensor of second order in relativistic transformation. (Figures S4-S6, Equation (S6)(S19)).

3) After encounter divergence problems in integrations, I can't help to separate Planck formula of thermal radiation into two parts; one is the wave noise and the other is the shot noise.

4) When the velocities of objects are comparable with light speed, the Newton's third law should be described as: The interactions or proper forces between two objects are equal and in opposite direction, but the visual forces are unequal in general.

\section{The Estimation about the Rest Mass of the Photon}

In the last century, the cosmic microwave background radiation [1] [2] was discovered and the cosmic background temperature $\left(T_{\mathrm{CB}}=2.725 \mathrm{~K}\right)$ is calculated by the data of COBE satellite [3]. Supposing every photon has the same rest mass $m_{\mathrm{s}}$ and the rest frequency $v_{\mathrm{s}}$, when it is moving with the group velocity $c_{\mathrm{g}}$, the parameters of the photon can be described as

$$
h v_{\mathrm{s}}=m_{\mathrm{s}} c^{2}, v=\gamma v_{\mathrm{s}}, c_{\mathrm{g}}^{2}=c^{2}\left(1-\gamma^{-2}\right) \text {. }
$$

In the thermal radiation of a black body, the intensity is composed of two parts: one is the shot noise and the other is the wave noise [4], and the Planck formula can be separated mathematically into two parts:

$$
\begin{aligned}
& I_{w}(f)=\frac{2 \pi c^{-2} h f^{3} \exp (-h f / k T)}{\exp (h f / k T)-1}, \\
& I_{\mathrm{p}}(v)=2 \pi c^{-2} h v^{3} \exp (-h v / k T) .
\end{aligned}
$$

Then the number density of the background photons at general temperature is

$$
n(v)=8 \pi c^{-3} C_{T} v^{2} \exp (-h v / k T) .
$$

When the temperature goes higher, the factor $C_{T}$ approaches to unit, and the distribution of photons agrees with Boson distribution at higher frequency but is different from that at lower frequency as the wave noise has been separated. Essentially, the difference is caused by photon's interaction which is rather strong for photons with the lower frequency and is weakened rapidly by the increase of the photon's frequency.

Considering the Faraday rotation effects, it is reasonable to suppose that every photon has the same proper magnetic moment, then the magnetic property of 
photon's rest energy will cause negative mechanical pressure in the photon gas, and the photon's kinetic energy will cause the positive one. The total mechanical pressure should be zero at the cosmic background temperature in the space where the photon gas is at an open state of thermal equilibrium. When the local temperature is higher than the cosmic background temperature, the mechanical pressure of the photon gas is positive and the energy will be diverged. However, when the local temperature is lower than the cosmic background temperature, the mechanical pressure of the photon gas is negative and the energy will be converged. At last, there is a stable cosmic background photon gas fulfilled in our universe. Supposing the average kinetic energy of the photons is equal to its rest energy at the cosmic background temperature, thus

$$
\int_{v_{\mathrm{s}}}^{\infty} h\left(v-2 v_{\mathrm{s}}\right) n(v) \mathrm{d} v=0, T=T_{\mathrm{CB}} .
$$

To calculate the integration (Equation (4), Equation (5)) and using the numerical method, the characteristic equation and its solution are

$$
a_{\mathrm{c}}^{3}+a_{\mathrm{c}}^{2}-2 a_{\mathrm{c}}-6=0, a_{\mathrm{c}}=h v_{\mathrm{s}} / k T_{\mathrm{CB}}=1.8454661 .
$$

Thus the mass and frequency of a rest photon are

$$
m_{\mathrm{s}}=7.725(3) \times 10^{-40} \mathrm{~kg}, \quad v_{\mathrm{s}}=1.0479(4) \times 10^{11} \mathrm{~Hz} .
$$

The interaction between photons forms the special frequency distribution and the unique density of temperature, then the photon gas is fluctuated in large scale, and its radiation property is the same as a black body at the equal temperature.

\section{The Magnetic-Electric Dynamics of Photon Gas}

If the average velocity of the photons is zero and the photon gas is an isotropic medium in visual system, then the photon gas is moving as an anisotropic medium in the proper system. Since the relativistic transformation of the magnetic moment is complicated as a tensor of second order, so that the magnetic field $\boldsymbol{B}$ should be defined based on the line current, then the interacting magnetic field is a tensor of second order in proper system (Figures S4-S6, Equations (S6)(S19)) and the magnetic inductivity is a tensor of second order in proper system too, but both the characteristic magnetic field and the characteristic magnetic inductivity are relatively simple in proper system.

A photon is traveling in the $y_{0}$ direction with the group velocity $c_{\mathrm{g}}$ in the visual system (Figure S7) and the suitable coordinates are selected. To simplify the mathematical analysis, considering one dimension electromagnetic wave $E_{y}-B_{z}$ in the visual system and the curl of the electromagnetic field can be calculated (Equations (S22)-(S25)).

The photon's proper magnetic moment can be equivalent to a circle current according to the principle of correspondence,

$$
\boldsymbol{m}_{\mathrm{p}}^{\prime}=\hat{x}_{0} m_{\mathrm{p} x_{0}}^{\prime}+\hat{y}_{0} m_{\mathrm{p} y_{0}}^{\prime}+\hat{z}_{0} m_{\mathrm{pz}_{0}}^{\prime} .
$$

The proper force acted on the photon by the curl of magnetic field has been 
analyzed (Figures S8-S11, Equation (S37), Equation (S44)) and can be calculated. The visual force acted on the photon has been calculated and the acceleration of the photon is (Equations (S38)-(S40)),

$$
\begin{aligned}
& \frac{\partial c_{\mathrm{gx}_{0}}}{\partial t}=\frac{\gamma^{-2} c^{2}}{h v_{\mathrm{s}}} \frac{\partial B_{z}}{\partial x} m_{\mathrm{pz}_{0}}^{\prime} \cos \theta_{y}, \\
& \frac{\partial c_{\mathrm{g}_{0}}}{\partial t}=\frac{\gamma^{-5} c^{2}}{h v_{\mathrm{s}}} \frac{\partial B_{z}}{\partial x} m_{\mathrm{pz}_{0}}^{\prime} \sin \theta_{y}, \\
& \frac{\partial c_{\mathrm{gz}_{0}}}{\partial t}=-\frac{\gamma^{-2} c^{2}}{h v_{\mathrm{s}}} \frac{\partial B_{z}}{\partial x}\left(m_{\mathrm{px}_{0}}^{\prime} \cos \theta_{y}+\gamma^{-1} m_{\mathrm{py}_{0}}^{\prime} \sin \theta_{y}\right) .
\end{aligned}
$$

The characteristic electric moment of a moving photon is (Figure S11, Equation (S45), Equation (S46))

$$
\boldsymbol{p}_{\mathrm{e}}=c^{-2} \gamma^{-2} c_{\mathrm{g}}\left(\hat{x}_{0} m_{\mathrm{pz}_{0}}^{\prime}-\hat{z}_{0} m_{\mathrm{p} \mathrm{x}_{0}}^{\prime}\right) .
$$

The partial derivatives of the characteristic electric moment of a moving photon with respect to photon's velocity are (Figure S12, Figure S13, Equations (S47)-(S51))

$$
\begin{aligned}
& \frac{\partial \boldsymbol{p}_{\mathrm{e}}}{\partial c_{\mathrm{g}_{0}}}=c^{-2} \gamma^{-2}\left(\hat{z}_{0} m_{\mathrm{p} y_{0}}^{\prime}-\hat{y}_{0} m_{\mathrm{p}_{0}}^{\prime}\right), \\
& \frac{\partial \boldsymbol{p}_{\mathrm{e}}}{\partial c_{\mathrm{g} y_{0}}}=c^{-2}\left(3 \gamma^{-2}-2\right)\left(\hat{x}_{0} m_{\mathrm{pz}_{0}}^{\prime}-\hat{z}_{0} m_{\mathrm{px}_{0}}^{\prime}\right), \\
& \frac{\partial \boldsymbol{p}_{\mathrm{e}}}{\partial c_{\mathrm{g}_{0}}}=c^{-2} \gamma^{-2}\left(\hat{y}_{0} m_{\mathrm{p} x_{0}}^{\prime}-\hat{x}_{0} m_{\mathrm{p} y_{0}}^{\prime}\right) .
\end{aligned}
$$

The partial derivative of the characteristic electric moment with respect to time can be calculated and averaged by the random properties of the photons in the state of thermal equilibrium (Equation (9), Equation (11), Equations (S52)(S55)), then

$$
\frac{\overline{\partial \boldsymbol{p}_{\mathrm{e}}}}{\partial t}=-\hat{y} \frac{2 m_{\mathrm{p}}^{\prime 2}}{9 h v_{\mathrm{s}}} \frac{\partial B_{z}}{\partial x}\left(\gamma^{-4}-\gamma^{-5}+3 \gamma^{-7}\right) .
$$

The electric field is the integration of the characteristic electric moment of the photons, and then the partial derivative of the electric field is

$$
\frac{\partial \boldsymbol{D}}{\partial t}=-\int_{1}^{\infty} \frac{\overline{\partial \boldsymbol{p}_{\mathrm{e}}}}{\partial t} n(\gamma) \mathrm{d} \gamma .
$$

To calculate the integration (Equation (4)) and defined function:

$$
K_{\varepsilon}=\frac{4}{5} \int_{1}^{\infty}\left(\gamma^{-2}-\gamma^{-3}+3 \gamma^{-5}\right) \exp \frac{-\gamma h v_{\mathrm{s}}}{k T} \mathrm{~d} \gamma .
$$

Then the magnetic-electric dynamic equation of the photon gas is

$$
\frac{\partial D_{y}}{\partial t}=\frac{20 \pi c^{-3} v_{\mathrm{s}}^{2} m_{\mathrm{p}}^{\prime 2}}{9 h} C_{T} K_{\varepsilon} \frac{\partial B_{z}}{\partial x} .
$$

The change of the electric field is counteractive compared with that in the Maxwell's equation, because the curl of magnetic field is active at the magne- 
to-dynamics of photon gas and is passive in the Maxwell's equation that is just the mechanism of Lenz law. At the high temperature and compared with the Maxwell's equation, the magnetic moment of a rest photon is (Equation (7))

$$
C_{T} K_{\varepsilon} \rightarrow 1, \quad m_{\mathrm{p}}^{\prime 2}=\frac{9 h c^{3}}{20 \pi v_{\mathrm{s}}^{2} \mu_{0}}, \quad m_{\mathrm{p}}^{\prime}=4.305(2) \times 10^{-13} \mathrm{~A} \cdot \mathrm{m}^{2} .
$$

Then the simplified magnetic-electric dynamic equation of photon gas at general temperature is

$$
\frac{\partial D_{y}}{\partial t}=\varepsilon_{0} c^{2} C_{T} K_{\varepsilon} \frac{\partial B_{z}}{\partial x}
$$

\section{The Electric-Magnetic Dynamics of Photon Gas}

A moving photon has two transverse components of characteristic electric moment and the longitudinal component is zero, since the transverse interaction is enlarged by one factor of relativity, so that the interacting electric moment is a tensor of second order. The first part of the proper force acted on the photon is the cross production of the interacting electric moment and the curl of electric field, and then the first part of the visual force is obtained (Equations (S56)-(S60)).

To transform the visual electric field into the characteristic magnetic field in the proper system and calculating its curl acted on the longitudinal proper magnetic moment of the photon (Equation (S26), Equation (S27)), then the second part of the visual force is obtained and the visual acceleration of the photon acted by the electric field is (Equations (S61)-(S65))

$$
\begin{aligned}
& \frac{\partial c_{\mathrm{g} x_{0}}}{\partial t}=\frac{\gamma^{-3} c_{\mathrm{g}}}{h v_{\mathrm{s}}} \frac{\partial E_{y}}{\partial x} m_{\mathrm{pg}_{0}}^{\prime} \sin \phi_{y} \sin \theta_{y}, \\
& \frac{\partial c_{\mathrm{g} y_{0}}}{\partial t}=\frac{\gamma^{-5} c_{\mathrm{g}}}{h v_{\mathrm{s}}} \frac{\partial E_{y}}{\partial x}\left(-m_{\mathrm{p}_{0}}^{\prime} \cos \phi_{y}-m_{\mathrm{p} x_{0}}^{\prime} \sin \phi_{y} \cos \theta_{y}\right), \\
& \frac{\partial c_{\mathrm{g}_{0}}}{\partial t}=\frac{\gamma^{-3} c_{\mathrm{g}}}{h v_{\mathrm{s}}} \frac{\partial E_{y}}{\partial x}\left(m_{\mathrm{pz}_{0}}^{\prime} \sin \phi_{y} \sin \theta_{y}+m_{\mathrm{p} y_{0}}^{\prime} \cos \phi_{y} \sin ^{2} \theta_{y}\right) .
\end{aligned}
$$

The characteristic magnetic moment of a moving photon in the visual system is (Figure S11, Equations (S41)-(S43))

$$
\boldsymbol{m}_{\mathrm{p}}=\hat{x}_{0} \gamma^{-2} m_{\mathrm{p} x_{0}}^{\prime}+\hat{y}_{0} \gamma^{-1} m_{\mathrm{p} y_{0}}^{\prime}+\hat{z}_{0} \gamma^{-2} m_{\mathrm{p} z_{0}}^{\prime} .
$$

The derivatives of the characteristic magnetic moment of a moving photon with respect to the photon's velocity are (Figure S14, Equations (S66)-(S70))

$$
\begin{aligned}
& \frac{\partial \boldsymbol{m}_{\mathrm{p}}}{\partial c_{\mathrm{g} x_{0}}}=c_{\mathrm{g}}^{-1}\left(\gamma^{-1}-\gamma^{-2}\right)\left(\hat{y}_{0} m_{\mathrm{p} x_{0}}^{\prime}+\hat{x}_{0} m_{\mathrm{p} y_{0}}^{\prime}\right), \\
& \frac{\partial \boldsymbol{m}_{\mathrm{p}}}{\partial c_{\mathrm{g}_{0}}}=c_{\mathrm{g}} c^{-2}\left(-\hat{x}_{0} 2 m_{\mathrm{p} x_{0}}^{\prime}-\hat{y}_{0} \gamma m_{\mathrm{p}_{0}}^{\prime}-\hat{z}_{0} 2 m_{\mathrm{pz}_{0}}^{\prime}\right), \\
& \frac{\partial \boldsymbol{m}_{\mathrm{p}}}{\partial c_{\mathrm{g}_{0}}}=c_{\mathrm{g}}^{-1}\left(\gamma^{-1}-\gamma^{-2}\right)\left(\hat{z}_{0} m_{\mathrm{p} y_{0}}^{\prime}+\hat{y}_{0} m_{\mathrm{pz}}^{\prime}\right) .
\end{aligned}
$$

The partial derivative of the characteristic magnetic moment with respect to 
the time can be calculated and averaged by the random properties of photons in the state of thermal equilibrium (Equation (18), Equation (20), Equation (S71), Equation (S72)), then

$$
\frac{\overline{\partial \boldsymbol{m}_{\mathrm{p}}}}{\partial t}=\hat{z} \frac{m_{\mathrm{p}}^{\prime 2}}{9 h v_{\mathrm{s}}} \frac{\partial E_{y}}{\partial x}\left(3 \gamma^{-4}+\gamma^{-5}-4 \gamma^{-7}\right) .
$$

The magnetic field is the integration of the characteristic magnetic moment of photons, and then the partial derivative of the magnetic field is

$$
\frac{\partial \boldsymbol{H}}{\partial t}=\int_{1}^{\infty} \frac{\overline{\partial \boldsymbol{m}_{\mathrm{p}}}}{\partial t} n(\gamma) \mathrm{d} \gamma .
$$

To calculate the integration (Equation (4)) and defined function:

$$
K_{\mu}=\frac{2}{5} \int_{1}^{\infty}\left(3 \gamma^{-2}+\gamma^{-3}-4 \gamma^{-5}\right) \exp \frac{-\gamma h v_{\mathrm{s}}}{k T} \mathrm{~d} \gamma
$$

Then the electric-magnetic dynamic equation of photon gas is

$$
\frac{\partial H_{z}}{\partial t}=\frac{20 \pi c^{-3} v_{\mathrm{s}}^{2} m_{\mathrm{p}}^{\prime 2}}{9 h} C_{T} K_{\mu} \frac{\partial E_{y}}{\partial x} .
$$

The change of the magnetic field is counteractive compared with that in the Maxwell's equation, because the curl of electric field is active at the electro-dynamics of photon gas and is passive in the Maxwell's equation that is the mechanism of Lenz law too. At high temperature and compared with the Maxwell's equation, the magnetic moment of rest photon has the same value as Eq. 16 , and the simplified electric-magnetic dynamic equation of the photon gas at general temperature is

$$
\frac{\partial H_{z}}{\partial t}=\mu_{0}^{-1} C_{T} K_{\mu} \frac{\partial E_{y}}{\partial x} .
$$

The wave equation can be obtained by the association of Equation (17) and Equation (25),

$$
\frac{\partial^{2} H_{z}}{\partial t^{2}}=c^{2} C_{T}^{2} K_{\varepsilon} K_{\mu} \frac{\partial^{2} H_{z}}{\partial x^{2}} .
$$

If the speed of electromagnetic wave is just equal to the ultimate velocity $\mathcal{c}$, in a good approximation, the temperature factor of the number density of photon gas is

$$
C_{T}=1 / \sqrt{K_{\varepsilon} K_{\mu}} .
$$

Then the three dimensional wave equation in photon gas is

$$
\partial^{2} \boldsymbol{H} / \partial t^{2}+c^{2} \nabla \times \nabla \times \boldsymbol{H}=0 .
$$

Since the electromagnetic field is discrete at the photon scale in photon gas, so that the two dynamic equations and the wave equation are only valued when the wavelength is far larger than the average interspaces of photons, and then the photon gas can be treated as a continuous medium.

\section{Conclusions and Discussions}

After the magnetic-electric and the electric-magnetic dynamic Equation (17), 
Equation (24) is established. It is clear that the photon gas is the carrier or medium of electromagnetic waves in vacuum and the main actor is the photons of lower velocity. The photon's velocity is a function of its visual frequency, after being separated from the ultimate speed $c$, the Einstein's special relativity is more solid. Since Equation (27) is only a good approximation, so that the speed of electromagnetic wave in photon gas should be a function of temperature too, and there should be a deviation, very small but not zero, between the ultimate speed $c$ and the wave speed at the cosmic background temperature. The photon's velocity will approach the ultimate speed when its visual frequency is very higher and the wave speed might approach the ultimate speed when the temperature of the photon gas is going higher too.

The propagation of electromagnetic wave is influenced by the moving of the photon gas, but the photon's traveling is rather independent to the moving of the photon gas in a short distance when its visual frequency is far higher than the rest one, so the null result had been got in the experiment of Michelson and Morley. When an electron and a photon are very nearby, their magnetic moments will entangle each other and that might be the process of Compton Effect, although the out behavior is like a collision.

\section{Acknowledgements}

I thank Dayou Ma, Peizi Lee and Zuomin Wang for their encouragement in 1979. I particularly thank Jianzhong Shen, Zhongcheng Liang and Yaling Yin for the useful discussions and the critical reading of the origin manuscript in 2009. I especially acknowledge Cornell University Library for their acceptance of my origin manuscript at Mar 2010 [5].

\section{References}

[1] Wilson, R.W. (1978) The Cosmic Microwave Background Radiation. Nobel Lecture.

[2] Penzias, A.A. (1978) The Origin of Elements. Nobel Lecture.

[3] Mather, J.C. (2006) From the Big Bang to Nobel Price and Beyond. Nobel Lecture.

[4] Smoot, G.F. (2006) Cosmic Microwave Background Radiation Anisotropies: Their Discovery and Utilization. Nobel Lecture.

[5] Chen, J.K. (2010) The Relativistic Dynamics of Photon Gas. Cornell University Library. http://arxiv.org/ftp/arxiv/papers/1003/1003.0255.pdf 


\section{Supporting Materials}

Method S1: Defined the Force Based on the Acceleration

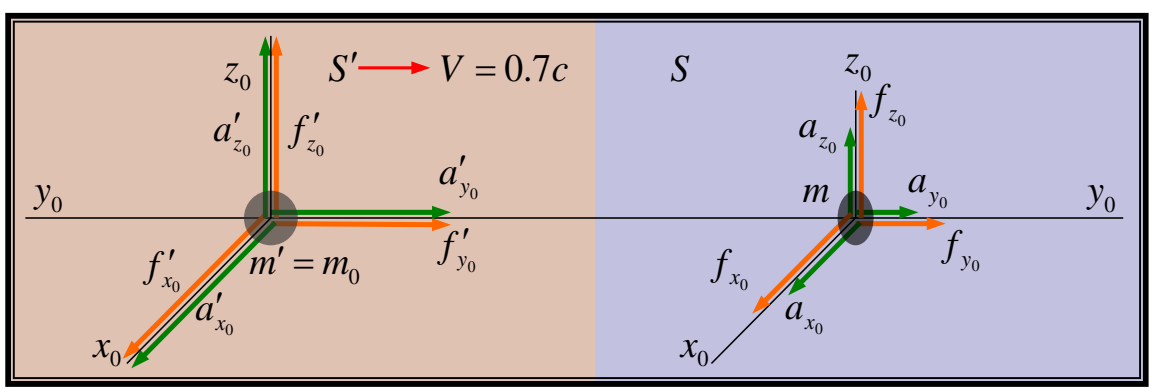

Figure S1. Transformations of acceleration.

Between the visual system $S$ where the observer is rest and the proper system $S^{\prime}$ where the object is rest, the transformations of acceleration and inertial mass are

$$
\begin{gathered}
a_{y_{0}}=\gamma^{-3} a_{y_{0}}^{\prime}, \quad a_{x_{0}, z_{0}}=\gamma^{-2} a_{x_{0}, z_{0}}^{\prime} \\
m=\gamma m^{\prime}=\gamma m_{0}
\end{gathered}
$$

Defined the force based on the acceleration, the transformations of the forces are

$$
f_{y_{0}}=\gamma^{-2} f_{y_{0}}^{\prime}, \quad f_{x_{0}, z_{0}}=\gamma^{-1} f_{x_{0}, z_{0}}^{\prime}
$$

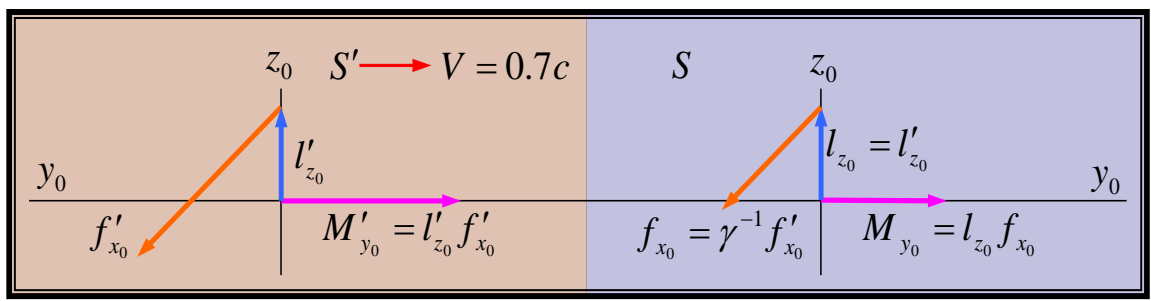

Figure S2. Longitudinal torque transformation.

The transformation of the longitudinal torque is

$$
M_{y_{0}}=\gamma^{-1} M_{y_{0}}^{\prime}
$$

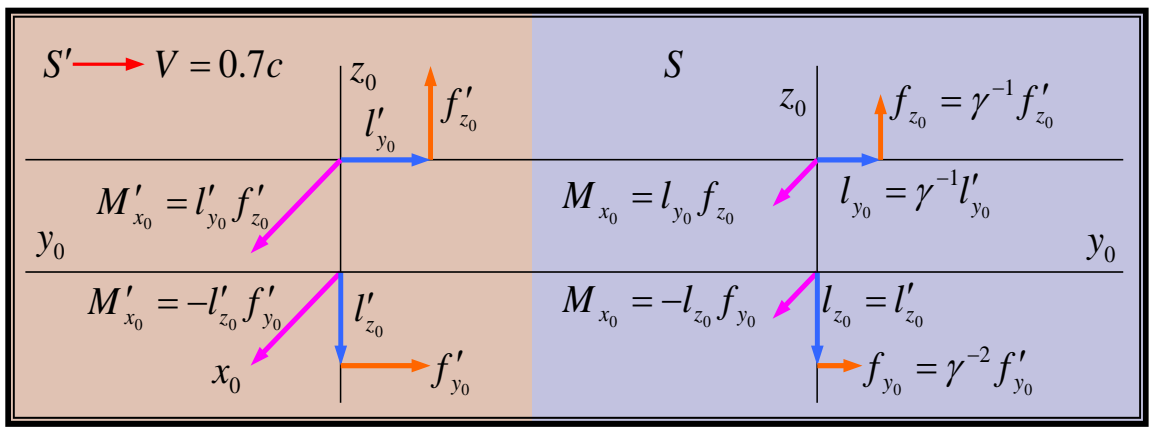

Figure S3. Transverse torques transformations. 
The transformations of the transverse torques are

$$
M_{x_{0}, z_{0}}=\gamma^{-2} M_{x_{0}, z_{0}}^{\prime}
$$

Method S2: Defined the Magnetic Field Based on the Line Current

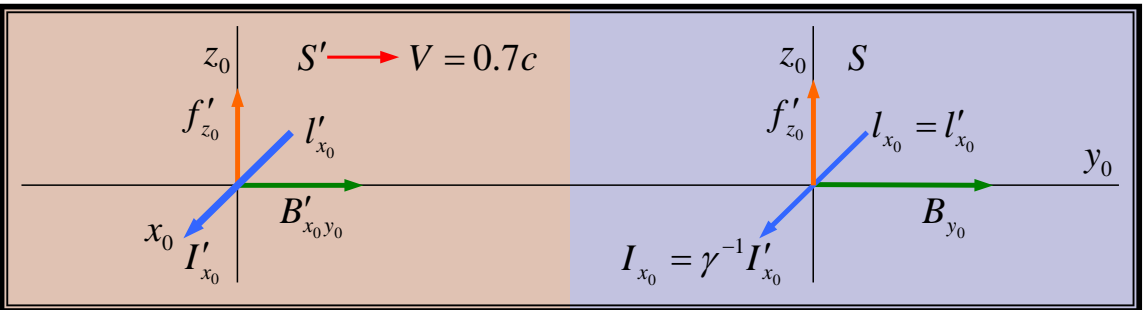

Figure S4. The definition of magnetic field based on the current.

To define the magnetic field $\boldsymbol{B}$ based on the line current. The relativistic transformations of the line currents are

$$
I_{y_{0}}=\gamma I_{y_{0}}^{\prime}, \quad I_{x_{0}, z_{0}}=\gamma^{-1} I_{x_{0}, z_{0}}^{\prime}
$$

In the visual system the interaction between the visual transverse current and the longitudinal magnetic field is $f_{z_{0}}^{\prime}=I_{x_{0}} l_{x_{0}} B_{y_{0}}$, in the proper system the interaction between the proper transverse current and the longitudinal interacting magnetic field is $f_{z_{0}}^{\prime}=I_{x_{0}}^{\prime} l_{x_{0}}^{\prime} B_{x_{0} y_{0}}^{\prime}$, then the transformations of the longitudinal magnetic field are

$$
\begin{aligned}
& B_{x_{0} y_{0}}^{\prime}=\gamma^{-1} B_{y_{0}} \\
& B_{z_{0} y_{0}}^{\prime}=\gamma^{-1} B_{y_{0}}
\end{aligned}
$$

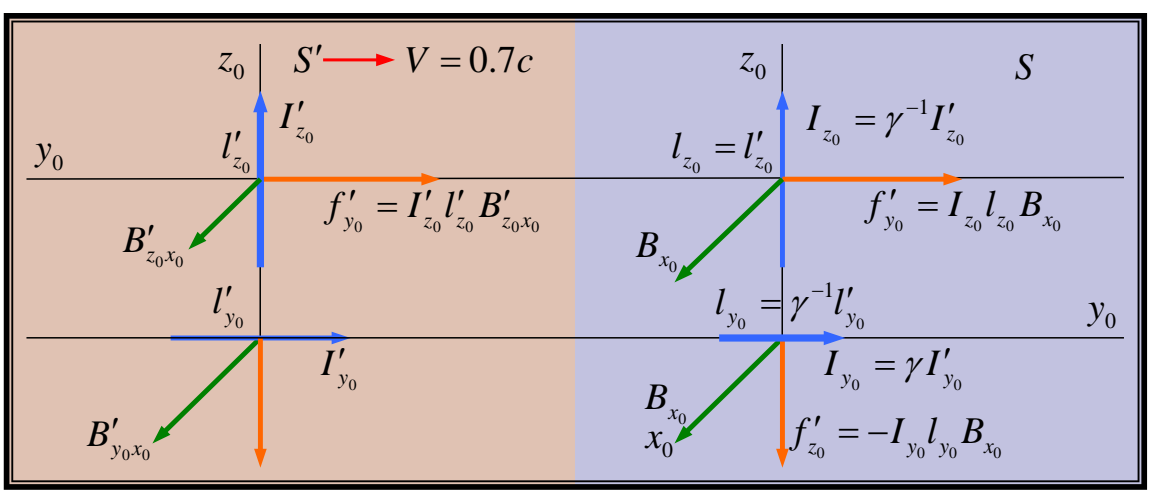

Figure S5. The interaction between the current and the magnetic field.

On the upper part of the figure the interaction between the visual transverse current and the transverse magnetic field is $f_{y_{0}}^{\prime}=I_{z_{0}} l_{z_{0}} B_{x_{0}}$, the interaction between the proper transverse current and the transverse interacting magnetic field is $f_{y_{0}}^{\prime}=I_{z_{0}}^{\prime} l_{z_{0}}^{\prime} B_{z_{0} x_{0}}^{\prime}$, then the transformations of the transverse magnetic field are

$$
\begin{aligned}
& B_{z_{0} x_{0}}^{\prime}=\gamma^{-1} B_{x_{0}} \\
& B_{x_{0} z_{0}}^{\prime}=\gamma^{-1} B_{z_{0}}
\end{aligned}
$$


On the lower part of the figure the interaction between the visual longitudinal current and the transverse magnetic field is $f_{z_{0}}^{\prime}=-I_{y_{0}} l_{y_{0}} B_{x_{0}}$, the interaction between the proper longitudinal current and the transverse interacting magnetic field is $f_{z_{0}}^{\prime}=-I_{y_{0}}^{\prime} l_{y_{0}}^{\prime} B_{y_{0} x_{0}}^{\prime}$, thus the transformations of the transverse magnetic field are

$$
\begin{aligned}
& B_{y_{0} x_{0}}^{\prime}=B_{x_{0}} \\
& B_{y_{0} z_{0}}^{\prime}=B_{z_{0}}
\end{aligned}
$$

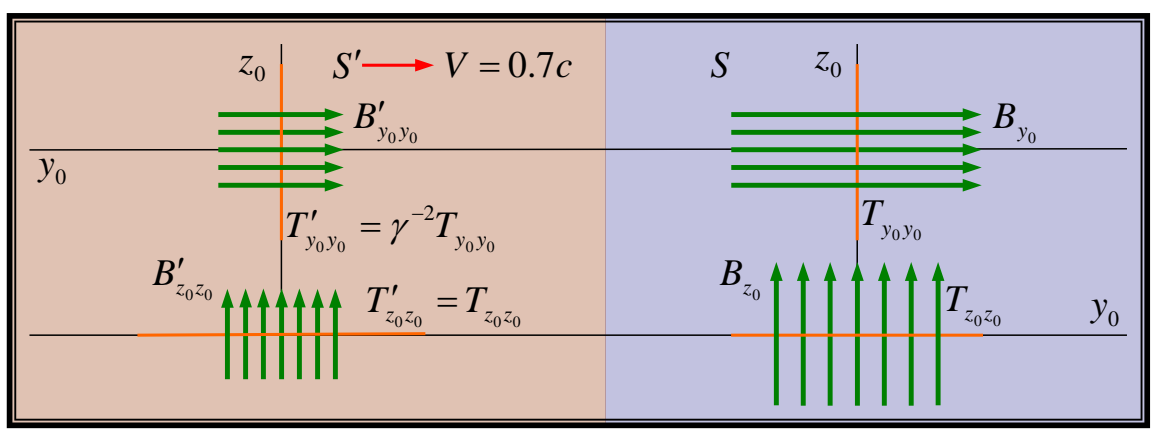

Figure S6. The characteristic magnetic field in proper system.

To determine the characteristic magnetic field in proper system, consider the magnetic field $B$ is a rest object in visual system and is a moving object in proper system, thus the transformations of the forces are the inversion of Equation (S3), and then the transformation of the longitudinal stress is

$$
T_{y_{0} y_{0}}^{\prime}=\gamma^{-2} T_{y_{0} y_{0}}, 2 T_{y_{0} y_{0}}^{\prime}=B_{y_{0}}^{\prime} H_{y_{0}}^{\prime}, 2 T_{y_{0} y_{0}}=B_{y_{0}} H_{y_{0}}, H_{y_{0}}^{\prime}=H_{y_{0}},
$$

and the transformation of the longitudinal characteristic magnetic field is

$$
B_{y_{0} y_{0}}^{\prime}=\gamma^{-2} B_{y_{0}}
$$

Similarly the transformations of the transverse characteristic magnetic field are

$$
\begin{aligned}
B_{z_{0} z_{0}}^{\prime} & =\gamma^{-1} B_{z_{0}} \\
B_{x_{0} x_{0}}^{\prime} & =\gamma^{-1} B_{x_{0}}
\end{aligned}
$$

It is clear that the interacting magnetic field is a tensor of second order (Equatins (S7)-(S15)) in the proper system:

$$
\left[\boldsymbol{B}^{\prime}\right]=B_{i_{0} j_{0}}^{\prime}=\left[\begin{array}{ccc}
B_{x_{0} x_{0}}^{\prime} & B_{x_{0} y_{0}}^{\prime} & B_{x_{0} z_{0}}^{\prime} \\
B_{y_{0} x_{0}}^{\prime} & B_{y_{0} y_{0}}^{\prime} & B_{y_{0} z_{0}}^{\prime} \\
B_{z_{0} x_{0}}^{\prime} & B_{z_{0} y_{0}}^{\prime} & B_{z_{0} z_{0}}^{\prime}
\end{array}\right]=\left[\begin{array}{ccc}
\gamma^{-1} B_{x_{0}} & \gamma^{-1} B_{y_{0}} & \gamma^{-1} B_{z_{0}} \\
B_{x_{0}} & \gamma^{-2} B_{y_{0}} & B_{z_{0}} \\
\gamma^{-1} B_{x_{0}} & \gamma^{-1} B_{y_{0}} & \gamma^{-1} B_{z_{0}}
\end{array}\right]
$$

The general transformation of the magnetic (potential) field is

$$
H_{x_{0}}^{\prime}=\gamma H_{x_{0}}, \quad H_{y_{0}}^{\prime}=H_{y_{0}}, \quad H_{z_{0}}^{\prime}=\gamma H_{z_{0}}
$$

Then in proper system the magnetic inductivity is a tensor of second order too

$$
\left[\boldsymbol{\mu}^{\prime}\right]=\mu_{i_{0} j_{0}}^{\prime}=\frac{B_{i_{0} j_{0}}^{\prime}}{H_{j_{0}}^{\prime}}=\mu_{0}\left[\begin{array}{lll}
\gamma^{-2} & \gamma^{-1} & \gamma^{-2} \\
\gamma^{-1} & \gamma^{-2} & \gamma^{-1} \\
\gamma^{-2} & \gamma^{-1} & \gamma^{-2}
\end{array}\right]=\gamma^{-2} \mu_{0}\left[\begin{array}{ccc}
1 & \gamma & 1 \\
\gamma & 1 & \gamma \\
1 & \gamma & 1
\end{array}\right]
$$


The characteristic magnetic field in the proper system is

$$
\boldsymbol{B}_{\mathrm{c}}^{\prime}=B_{i_{0}}^{\prime}=B_{i_{0} i_{0}}^{\prime}=\hat{x}_{0} \gamma^{-1} B_{x_{0}}+\hat{y}_{0} \gamma^{-2} B_{y_{0}}+\hat{z}_{0} \gamma^{-1} B_{z_{0}}
$$

The characteristic magnetic inductivity in the proper system is

$$
\mu_{\mathrm{c}}^{\prime}=\gamma^{-2} \mu_{0}
$$

\section{Method S3: The Coordinate Transformation for the Moving} Photon

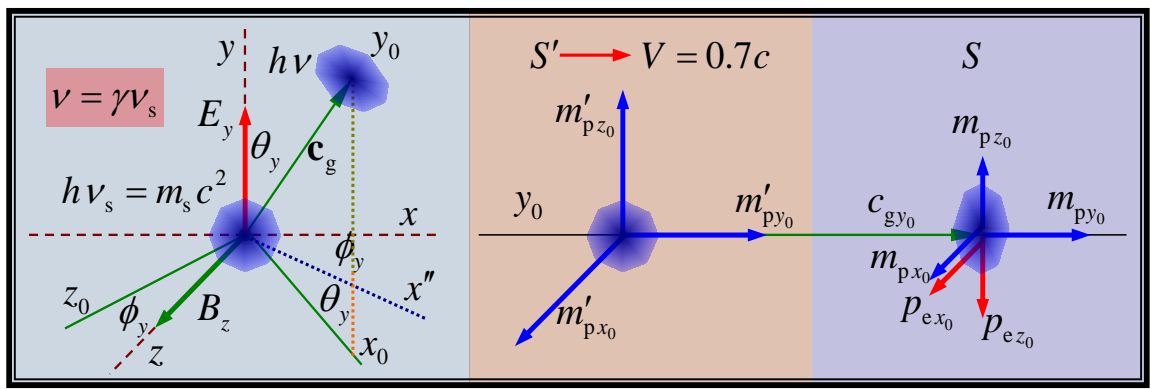

Figure S7. Spherical coordinate transformations of a moving photon.

When a photon is moving in the $y_{0}$ direction, the coordinate transformations are

$$
\begin{aligned}
& {\left[\begin{array}{c}
\hat{x} \\
\hat{y} \\
\hat{z}
\end{array}\right]=\left[\begin{array}{ccc}
\cos \theta_{y} \cos \phi_{y} & \sin \theta_{y} \cos \phi_{y} & -\sin \phi_{y} \\
-\sin \theta_{y} & \cos \theta_{y} & 0 \\
\cos \theta_{y} \sin \phi_{y} & \sin \theta_{y} \sin \phi_{y} & \cos \phi_{y}
\end{array}\right]\left[\begin{array}{l}
\hat{x}_{0} \\
\hat{y}_{0} \\
\hat{z}_{0}
\end{array}\right]} \\
& {\left[\begin{array}{c}
\hat{x}_{0} \\
\hat{y}_{0} \\
\hat{z}_{0}
\end{array}\right]=\left[\begin{array}{ccc}
\cos \theta_{y} \cos \phi_{y} & -\sin \theta_{y} & \cos \theta_{y} \sin \phi_{y} \\
\sin \theta_{y} \cos \phi_{y} & \cos \theta_{y} & \sin \theta_{y} \sin \phi_{y} \\
-\sin \phi_{y} & 0 & \cos \phi_{y}
\end{array}\right]\left[\begin{array}{c}
\hat{x} \\
\hat{y} \\
\hat{z}
\end{array}\right]}
\end{aligned}
$$

To simplify the mathematical analysis, considering one dimension electromagnetic wave $E_{y}-B_{z}$, in the visual system the curl of magnetic field is,

$$
\operatorname{curl} \boldsymbol{B}=-\hat{y} \partial B_{z} / \partial x=\left(\hat{x}_{0} \sin \theta_{y}-\hat{y}_{0} \cos \theta_{y}\right) \partial B_{z} / \partial x
$$

In the visual system the curl of electric field is

$$
\operatorname{curl} \boldsymbol{E}=\left(\hat{x}_{0} \sin \phi_{y} \cos \theta_{y}+\hat{y}_{0} \sin \phi_{y} \sin \theta_{y}+\hat{z}_{0} \cos \phi_{y}\right) \partial E_{y} / \partial x
$$

Using Equation (S18), the transformations of the curls of magnetic field are

$$
\begin{aligned}
& \operatorname{curl}_{x_{0}}^{\prime} \boldsymbol{B}_{\mathrm{c}}^{\prime}=\gamma^{-2} \operatorname{curl}_{x_{0}} \boldsymbol{B}, \\
& \operatorname{curl}_{y_{0}}^{\prime} \boldsymbol{B}_{\mathrm{c}}^{\prime}=\gamma^{-1} \operatorname{curl}_{y_{0}} \boldsymbol{B}, \\
& \operatorname{curl}_{z_{0}}^{\prime} \boldsymbol{B}_{\mathrm{c}}^{\prime}=\gamma^{-2} \operatorname{curl}_{z_{0}} \boldsymbol{B} .
\end{aligned}
$$

Then in the proper system the curl of magnetic field is (Equation (S22), Equation (S24)),

$$
\operatorname{curl}^{\prime} \boldsymbol{B}_{\mathrm{c}}^{\prime}=\left(\hat{x}_{0} \gamma^{-2} \sin \theta_{y}-\hat{y}_{0} \gamma^{-1} \cos \theta_{y}\right) \partial B_{z} / \partial x
$$

To transform the visual electric field $E_{y}$ into the characteristic magnetic field 
in the proper system

$$
\boldsymbol{B}_{\mathrm{c}, \boldsymbol{E}}^{\prime}=\mu_{\mathrm{c}}^{\prime} \boldsymbol{H}_{\boldsymbol{E}}^{\prime}=\gamma^{-2} \mu_{0} \boldsymbol{H}_{\boldsymbol{E}}^{\prime}=-\gamma^{-1} c^{-2} \boldsymbol{c}_{\mathrm{g}} \times \boldsymbol{E}=-\hat{z}_{0} \gamma^{-1} c^{-2} c_{\mathrm{g}} \sin \theta_{y} E_{y}
$$

For one dimension wave in the $x$ direction (Equation (S21))

$$
\begin{gathered}
\frac{\partial E_{y}}{\partial y}=0, \quad \frac{\partial E_{y}}{\partial z}=0, \quad \frac{\partial E_{y}}{\partial x_{0}^{\prime}}=\cos \phi_{y} \cos \theta_{y} \frac{\partial E_{y}}{\partial x}, \\
\frac{\partial E_{y}}{\partial y_{0}^{\prime}}=\gamma^{-1} \cos \phi_{y} \sin \theta_{y} \frac{\partial E_{y}}{\partial x}, \quad \frac{\partial E_{y}}{\partial z_{0}^{\prime}}=-\sin \phi_{y} \frac{\partial E_{y}}{\partial x} . \\
\frac{\operatorname{curl}^{\prime} \boldsymbol{B}_{\mathrm{c}, \boldsymbol{E}}^{\prime}}{\partial E_{y} / \partial x}=-c^{-2} c_{\mathrm{g}}\left(\hat{x}_{0} \gamma^{-2} \cos \phi_{y} \sin ^{2} \theta_{y}-\hat{y}_{0} \gamma^{-1} \cos \phi_{y} \sin \theta_{y} \cos \theta_{y}\right) .
\end{gathered}
$$

\section{Method S4: The Curl of Magnetic Field Interacts with the Magnetic Moment}

If every photon has a same proper magnetic moment, then in the visual system the moving photon has three components of characteristic magnetic moment and two transverse components of characteristic electric moment.

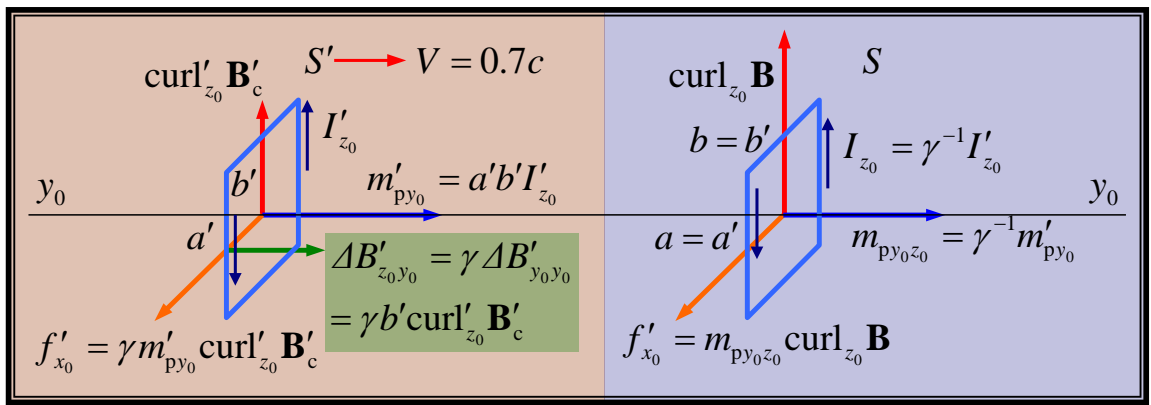

Figure S8. The curl of magnetic field interacts with the magnetic moment (1).

The transverse curl of magnetic field interacts with the longitudinal magnetic moment. In the proper system the transverse curl is equivalent to an increment of magnetic field which interacted on one edgy of the longitudinal magnetic moment (Equatiom (S16), Equatiom (S24)),

$$
\Delta B_{z_{0} y_{0}}^{\prime}=\gamma \Delta B_{y_{0} y_{0}}^{\prime}=\gamma^{-1} b^{\prime} \operatorname{curl}_{z_{0}} \boldsymbol{B}_{\mathrm{c}}
$$

The proper force acted on the proper magnetic moment is

$$
f_{x_{0}}^{\prime}=I_{z_{0}}^{\prime} a^{\prime} \Delta B_{z_{0} y_{0}}^{\prime}=\gamma^{-1} m_{\mathrm{p} y_{0}}^{\prime} \operatorname{curl}_{z_{0}} \boldsymbol{B}
$$

In the visual system one component of the visual magnetic moment is defined as

$$
\begin{gathered}
f_{x_{0}}^{\prime}=m_{\mathrm{p} y_{0} z_{0}} \operatorname{curl}_{z_{0}} \boldsymbol{B} \text {, then } \\
m_{\mathrm{p} y_{0} z_{0}}=\gamma^{-1} m_{\mathrm{p} y_{0}}^{\prime} \\
m_{\mathrm{p} y_{0} x_{0}}=\gamma^{-1} m_{\mathrm{p} y_{0}}^{\prime}
\end{gathered}
$$

The proper force acted on the longitudinal magnetic moment by the transverse curl of magnetic field can be written in the form of determinant as 


$$
f_{B 1}^{\prime}=\left|\begin{array}{ccc}
\hat{x}_{0} & C & \hat{z}_{0} \\
0 & \gamma^{-1} m_{\mathrm{p} y_{0}}^{\prime} & 0 \\
\operatorname{curl}_{x_{0}} \boldsymbol{B} & 0 & \operatorname{curl}_{z_{0}} \boldsymbol{B}
\end{array}\right|_{S}=\left|\begin{array}{ccc}
\hat{x}_{0} \gamma & C & \hat{z}_{0} \gamma \\
0 & m_{\mathrm{p} y_{0}}^{\prime} & 0 \\
\operatorname{curl}_{x_{0}}^{\prime} \boldsymbol{B}_{\mathrm{c}}^{\prime} & 0 & \operatorname{curl}_{z_{0}}^{\prime} \boldsymbol{B}_{\mathrm{c}}^{\prime}
\end{array}\right|_{S^{\prime}}
$$

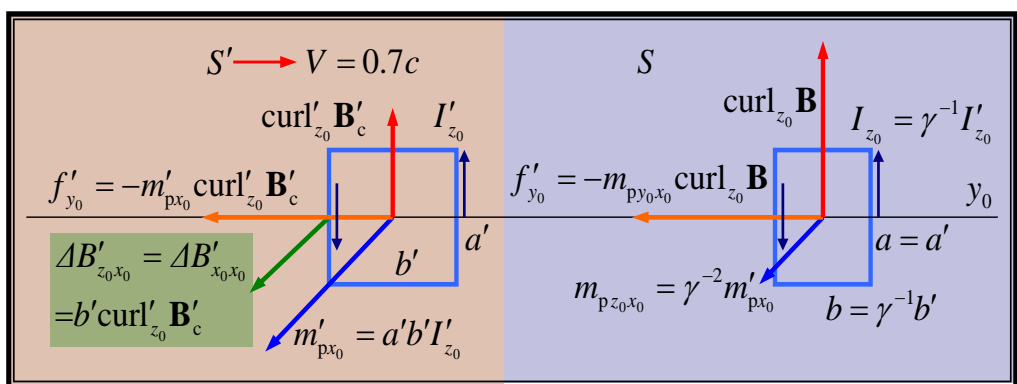

Figure S9. The curl of magnetic field interacts with the magnetic moment (2).

The transverse curl of magnetic field interacts with the transverse magnetic moment. In the proper system the transverse curl is equivalent to an increment of magnetic field which interacted on one edgy of the transverse magnetic moment (Equation (S16), Equation (S24)),

$$
\Delta B_{z_{0} x_{0}}^{\prime}=\Delta B_{x_{0} x_{0}}^{\prime}=\gamma^{-2} b^{\prime} \operatorname{curl}_{z_{0}} \boldsymbol{B}_{\mathrm{c}}
$$

The proper force acted on the proper magnetic moment is

$$
f_{y_{0}}^{\prime}=-I_{z_{0}}^{\prime} a^{\prime} \Delta B_{z_{0} x_{0}}^{\prime}=-\gamma^{-2} m_{\mathrm{p} x_{0}}^{\prime} \operatorname{curl}_{z_{0}} B
$$

In visual system one component of the visual magnetic moment is defined as

$$
\begin{gathered}
f_{y_{0}}^{\prime}=-m_{\mathrm{p} x_{0} z_{0}} \operatorname{curl}_{z_{0}} \boldsymbol{B} \text {, then } m_{\mathrm{p} x_{0} z_{0}}=\gamma^{-2} m_{\mathrm{p} x_{0}}^{\prime} \\
\text { and } m_{\mathrm{p} z_{0} x_{0}}=\gamma^{-2} m_{\mathrm{p} z_{0}}^{\prime}
\end{gathered}
$$

The proper force acted on the transverse magnetic moment by the transverse curl of magnetic field can be written in the form of determinant as

$$
f_{B 2}^{\prime}=\left|\begin{array}{ccc}
C & \hat{y}_{0} \gamma^{-2} & C \\
m_{\mathrm{p} x_{0}}^{\prime} & 0 & m_{\mathrm{p} z_{0}}^{\prime} \\
\operatorname{curl}_{x_{0}} \boldsymbol{B} & 0 & \operatorname{curl}_{z_{0}} \boldsymbol{B}
\end{array}\right|_{S}=\left|\begin{array}{ccc}
C & \hat{y}_{0} & C \\
m_{\mathrm{p} x_{0}}^{\prime} & 0 & m_{\mathrm{pz}}^{\prime} \\
\operatorname{curl}_{x_{0}}^{\prime} \boldsymbol{B}_{\mathrm{c}}^{\prime} & 0 & \operatorname{curl}_{z_{0}}^{\prime} \boldsymbol{B}_{\mathrm{c}}^{\prime}
\end{array}\right|_{S^{\prime}}
$$

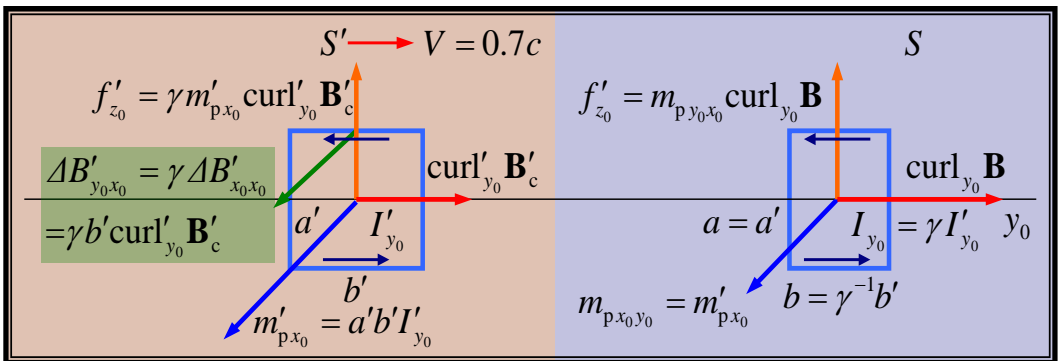

Figure S10. The curl of magnetic field interacts with the magnetic moment (3). 
The longitudinal curl of magnetic field interacts with the transverse magnetic moment. In proper system the longitudinal curl is equivalent to an increment of magnetic field which interacted on one edgy of the transverse magnetic moment (Equation (S16), Equation (S24)),

$$
\Delta B_{y_{0} x_{0}}^{\prime}=\gamma \Delta B_{x_{0} x_{0}}^{\prime}=b^{\prime} \operatorname{curl}_{z_{0}} B
$$

The proper force acted on the proper magnetic moment is

$$
f_{z_{0}}^{\prime}=I_{y_{0}}^{\prime} a^{\prime} \Delta B_{y_{0} x_{0}}^{\prime}=m_{\mathrm{p} x_{0}}^{\prime} \operatorname{curl}_{y_{0}} \boldsymbol{B}
$$

In visual system one component of the visual magnetic moment is defined as

$$
\begin{aligned}
& \quad f_{z_{0}}^{\prime}=m_{\mathrm{p} x_{0} y_{0}} \operatorname{curl}_{y_{0}} \boldsymbol{B}, \\
& \text { then } m_{\mathrm{p} x_{0} y_{0}}=m_{\mathrm{p} x_{0}}^{\prime} \\
& \text { and } m_{\mathrm{pz}_{0} y_{0}}=m_{\mathrm{p} z_{0}}^{\prime}
\end{aligned}
$$

The proper force acted on the transverse magnetic moment by the longitudinal curl of magnetic field can be written in the form of determinant as

$$
m_{\mathrm{pz}_{0} y_{0}}=m_{\mathrm{p} z_{0}}^{\prime} f_{B 3}^{\prime}=\left|\begin{array}{ccc}
\hat{x}_{0} & C & \hat{z}_{0} \\
m_{\mathrm{p} x_{0}}^{\prime} & 0 & m_{\mathrm{pz}}^{\prime} \\
0 & \operatorname{curl}_{y_{0}} \boldsymbol{B} & 0
\end{array}\right|_{S}=\left|\begin{array}{ccc}
\hat{x}_{0} \gamma & C & \hat{z}_{0} \gamma \\
m_{\mathrm{p} x_{0}}^{\prime} & 0 & m_{\mathrm{pz}_{0}}^{\prime} \\
0 & \operatorname{curl}_{y_{0}}^{\prime} \boldsymbol{B}_{\mathrm{c}}^{\prime} & 0
\end{array}\right|_{S^{\prime}}
$$

The proper force acted on the photon by the curl of magnetic field (Equation (S8), Equations (S30), (S33) and (S36)) is

$$
f_{B}^{\prime}=\left|\begin{array}{ccc}
\hat{x}_{0} & \hat{y}_{0} \gamma^{-2} & \hat{z}_{0} \\
m_{\mathrm{p} x_{0}}^{\prime} & \gamma^{-1} m_{\mathrm{p}_{0}}^{\prime} & m_{\mathrm{pz}_{0}}^{\prime} \\
\operatorname{curl}_{x_{0}} \boldsymbol{B} & \operatorname{curl}_{y_{0}} \boldsymbol{B} & \operatorname{curl}_{z_{0}} \boldsymbol{B}
\end{array}\right|_{S}=\left|\begin{array}{ccc}
\hat{x}_{0} \gamma & \hat{y}_{0} & \hat{z}_{0} \gamma \\
m_{\mathrm{p}_{0}}^{\prime} & m_{\mathrm{py}_{0}}^{\prime} & m_{\mathrm{p} z_{0}}^{\prime} \\
\operatorname{curl}_{x_{0}}^{\prime} \boldsymbol{B}^{\prime} & \operatorname{curl}_{y_{0}}^{\prime} \boldsymbol{B}^{\prime} & \operatorname{curl}_{z_{0}}^{\prime} \boldsymbol{B}^{\prime}
\end{array}\right|_{S^{\prime}}
$$

The interaction between the moving photon and the magnetic field is complicated by the tensor property of the interacting magnetic moment of the moving photon in the visual system, and is complicated by the tensor property of the interacting magnetic field in the proper system where the photon gas is moving as an anisotropic medium.

The visual force acted on the photon (Equation (S3), (S22) and (S37)) by the curl of magnetic field is

$$
\begin{gathered}
f_{B}=\left|\begin{array}{ccc}
\hat{x}_{0} \gamma^{-1} & \hat{y}_{0} \gamma^{-4} & \hat{z}_{0} \gamma^{-1} \\
m_{\mathrm{p} x_{0}}^{\prime} & \gamma^{-1} m_{\mathrm{py}_{0}}^{\prime} & m_{\mathrm{p} z_{0}}^{\prime} \\
\operatorname{curl}_{x_{0}} \boldsymbol{B} & \operatorname{curl}_{y_{0}} \boldsymbol{B} & \operatorname{curl}_{z_{0}} \boldsymbol{B}
\end{array}\right|=\left|\begin{array}{ccc}
\hat{x}_{0} & \hat{y}_{0} \gamma^{-2} & \hat{z}_{0} \\
m_{\mathrm{p} x_{0}}^{\prime} & m_{\mathrm{p} y_{0}}^{\prime} & m_{\mathrm{p}_{0}}^{\prime} \\
\operatorname{curl}_{x_{0}}^{\prime} \boldsymbol{B}^{\prime} & \operatorname{curl}_{y_{0}}^{\prime} \boldsymbol{B}^{\prime} & \operatorname{curl}_{\mathrm{z}_{0}}^{\prime} \boldsymbol{B}^{\prime}
\end{array}\right| \\
\frac{f_{B}}{\partial B_{z} / \partial x}=\left(\begin{array}{c}
\hat{x}_{0} \gamma^{-1} m_{\mathrm{pz}_{0}}^{\prime} \cos \theta_{y} \\
\hat{y}_{0} \gamma^{-4} m_{\mathrm{p} z_{0}}^{\prime} \sin \theta_{y} \\
\hat{z}_{0} \gamma^{-1}\left(-m_{\mathrm{p} x_{0}}^{\prime} \cos \theta_{y}-\gamma^{-1} m_{\mathrm{py}}^{\prime} \sin \theta_{y}\right)
\end{array}\right)
\end{gathered}
$$

Then the acceleration of the photon acted by the curl of magnetic field is 


$$
\left[\begin{array}{c}
\partial c_{\mathrm{gx}_{0}} / \partial t \\
\partial c_{\mathrm{g}_{0}} / \partial t \\
\partial c_{\mathrm{gz}_{0}} / \partial t
\end{array}\right]_{B}=\frac{\gamma^{-2} c^{2} \partial B_{z} / \partial x}{h v_{\mathrm{s}}}\left[\begin{array}{c}
m_{\mathrm{pz}_{0}}^{\prime} \cos \theta_{y} \\
\gamma^{-3} m_{\mathrm{pz}_{0}}^{\prime} \sin \theta_{y} \\
-m_{\mathrm{p} x_{0}}^{\prime} \cos \theta_{y}-\gamma^{-1} m_{\mathrm{py}_{0}}^{\prime} \sin \theta_{y}
\end{array}\right]
$$

Method S5: The Characteristic Moment of a Moving Photon

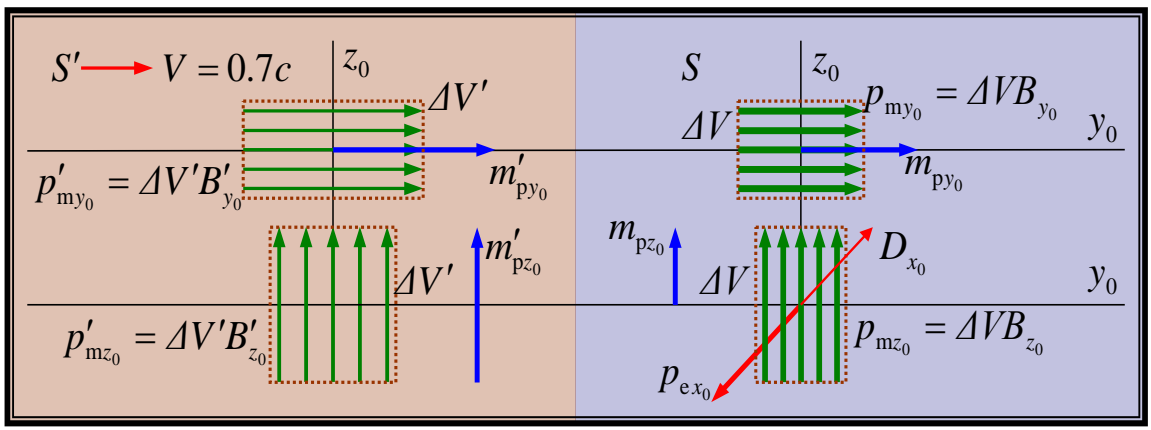

Figure S11. The characteristic magnetic moments of a moving photon.

The characteristic moments of a moving photon can be deduced from the characteristic magnetic field. On the upper part of the figure, the longitudinal magnetic dipole moment is $p_{\mathrm{m} y_{0}}^{\prime}=\gamma^{-2} \mu_{0} m_{\mathrm{p} y_{0}}^{\prime}=\Delta V^{\prime} B_{y_{0}}^{\prime}$ in the proper system, and is $p_{\mathrm{m} y_{0}}=\mu_{0} m_{\mathrm{p} y_{0}}=\Delta V B_{y_{0}}$ in the visual system. The transformation of the magnetic field is $B_{y_{0}}=\gamma^{2} B_{y_{0}}^{\prime}$ (Equation (S18)), and the transformation of the volume is $\Delta V=\gamma^{-1} \Delta V^{\prime}$, and then the longitudinal characteristic magnetic moment is

$$
m_{\mathrm{p} y_{0}}=\gamma^{-1} m_{\mathrm{p} y_{0}}^{\prime}
$$

On the lower part of the figure, the transverse magnetic dipole moment is $p_{\mathrm{m}_{0}}^{\prime}=\gamma^{-2} \mu_{0} m_{\mathrm{pz}_{0}}^{\prime}=\Delta V^{\prime} B_{z_{0}}^{\prime}$ in the proper system, and is $p_{\mathrm{m}_{0}}=\mu_{0} m_{\mathrm{p} z_{0}}=\Delta V B_{z_{0}}$ in the visual system. The transformations of the magnetic field and the volume are $B_{z_{0}}=\gamma B_{z_{0}}^{\prime}$ (Equation (S18)) and $\Delta V=\gamma^{-1} \Delta V^{\prime}$, then the transverse characteristic magnetic moments are

$$
m_{\mathrm{p} x_{0}}=\gamma^{-2} m_{\mathrm{p} x_{0}}^{\prime}, \quad m_{\mathrm{p} z_{0}}=\gamma^{-2} m_{\mathrm{p} z_{0}}^{\prime}
$$

The interacting magnetic moment of a moving photon is a tensor of second order in the visual system (Equations (S28), (S29), (S31), (S32), (S34), (S35), (S41) and (S42)),

$$
m_{\mathrm{pi}_{0} j_{0}}=\left[\begin{array}{ccc}
\gamma^{-2} m_{\mathrm{px_{0 }}}^{\prime} & m_{\mathrm{px}_{0}}^{\prime} & \gamma^{-2} m_{\mathrm{px_{0 }}}^{\prime} \\
\gamma^{-1} m_{\mathrm{py} y_{0}}^{\prime} & \gamma^{-1} m_{\mathrm{py}_{0}}^{\prime} & \gamma^{-1} m_{\mathrm{py}}^{\prime} \\
\gamma^{-2} m_{\mathrm{pz}_{0}}^{\prime} & m_{\mathrm{pz_{0 }}}^{\prime} & \gamma^{-2} m_{\mathrm{pz_{0 }}}^{\prime}
\end{array}\right]
$$

The proper force acted on the photon by the curl of magnetic field is the cross production of the magnetic moment and the curl of magnetic field in the visual system: (equivalent to Equation (37))

$$
\left[\begin{array}{c}
f_{x_{0}}^{\prime} \\
f_{y_{0}}^{\prime} \\
f_{z_{0}}^{\prime}
\end{array}\right]=\left[\begin{array}{l}
m_{\mathrm{py}_{0} z_{0}} \operatorname{curl}_{z_{0}} \boldsymbol{B}-m_{\mathrm{pz}_{0} y_{0}} \operatorname{curl}_{y_{0}} \boldsymbol{B} \\
m_{\mathrm{pz}_{0} x_{0}} \operatorname{curl}_{x_{0}} \boldsymbol{B}-m_{\mathrm{px}_{0} z_{0}} \operatorname{curl}_{z_{0}} \boldsymbol{B} \\
m_{\mathrm{px}_{0} y_{0}} \operatorname{curl}_{y_{0}} \boldsymbol{B}-m_{\mathrm{py}_{0} x_{0}} \operatorname{curl}_{x_{0}} \boldsymbol{B}
\end{array}\right]_{S}
$$


The moving photon has two characteristic electric moments, illustrated in the lower part of the figure,

$$
\begin{gathered}
p_{\mathrm{ex}_{0}}=-D_{x_{0}} \Delta V=\varepsilon_{0} c_{\mathrm{g}} p_{\mathrm{m}_{0}}^{\prime}=c^{-2} c_{\mathrm{g}} \gamma^{-2} m_{\mathrm{p} z_{0}}^{\prime} \\
p_{\mathrm{ez}_{0}}=-c^{-2} c_{\mathrm{g}} \gamma^{-2} m_{\mathrm{p} x_{0}}^{\prime}
\end{gathered}
$$

Method S6: The Derivative of the Characteristic Electric Moment of the Photon

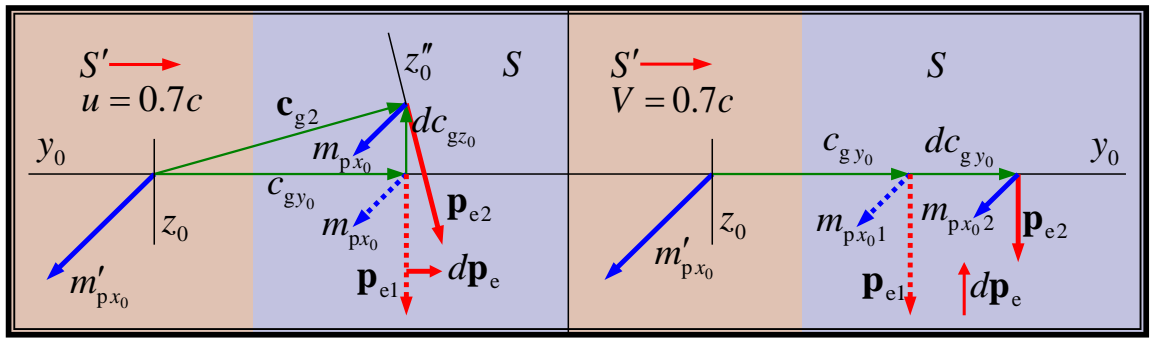

Figure S12. The characteristic electric moments of a moving photon.

The characteristic electric moment of a transverse magnetic moment is changed by the acceleration of the photon. On the left of the figure (Equation S46)

$$
\boldsymbol{p}_{\mathrm{e} 1}=-\hat{\mathrm{z}}_{0} c^{-2} c_{\mathrm{g} y_{0}} \gamma^{-2} m_{\mathrm{p} x_{0}}^{\prime}, \boldsymbol{p}_{\mathrm{e} 2}=-\hat{\mathrm{z}}_{0}^{\prime \prime} c^{-2} c_{\mathrm{g} y_{0}} \gamma^{-2} m_{\mathrm{p} x_{0}}^{\prime},
$$

and the derivative of the characteristic electric moment is

$$
\mathrm{d} \boldsymbol{p}_{\mathrm{e}}=\boldsymbol{p}_{\mathrm{e} 2}-\boldsymbol{p}_{\mathrm{e} 1}=\hat{y}_{0} c^{-2} \gamma^{-2} m_{\mathrm{px}_{0}}^{\prime} \mathrm{d} c_{\mathrm{gz}_{0}}
$$

On the right of the figure, the derivative of the characteristic electric moment can be calculated directly (Equation (S46)),

$$
\mathrm{d} \boldsymbol{p}_{\mathrm{e}}=-\hat{z}_{0} c^{-2}\left(3 \gamma^{-2}-2\right) m_{\mathrm{p} x_{0}}^{\prime} \mathrm{d} c_{g y_{0}}
$$

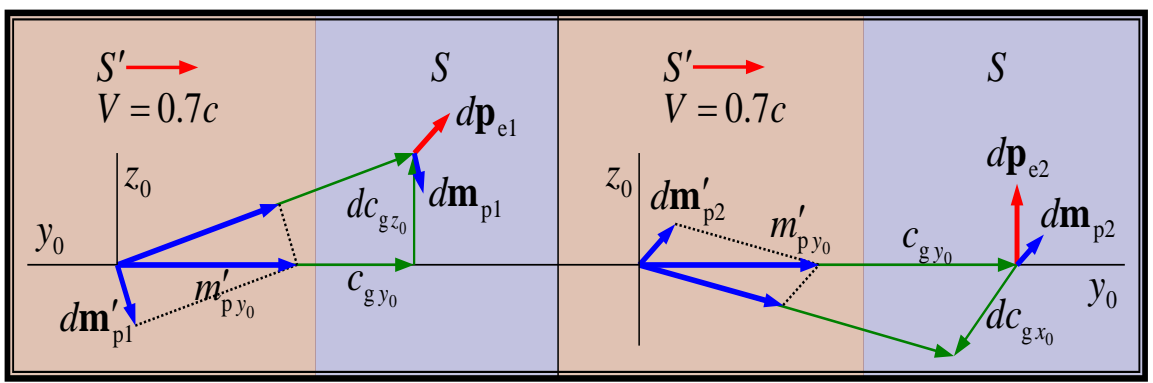

Figure S13. The change of the characteristic electric moment.

The characteristic electric moment of a longitudinal magnetic moment is changed by the acceleration of the photon. On the left of the Figure (Equation (S19)),

$$
\begin{aligned}
& \mathrm{d} \boldsymbol{m}_{\mathrm{p} 1}^{\prime}=-\hat{\mathrm{z}}_{0} c_{\mathrm{g}_{0}}^{-1} m_{\mathrm{py}}^{\prime} \mathrm{d} c_{\mathrm{gz}_{0}}, \\
& \mathrm{~d} \boldsymbol{p}_{\mathrm{e} 1}=c^{-2} \gamma^{-2} \boldsymbol{c}_{\mathrm{g}} \times \mathrm{d} \boldsymbol{m}_{\mathrm{p} 1}^{\prime}=-\hat{x}_{0} c^{-2} \gamma^{-2} m_{\mathrm{py}}^{\prime} \mathrm{d} c_{\mathrm{g} z_{0}}
\end{aligned}
$$


On the right of the Figure,

$$
\begin{aligned}
& \mathrm{d} \boldsymbol{m}_{\mathrm{p} 2}^{\prime}=-\hat{x}_{0} c_{\mathrm{g}}^{-1} m_{\mathrm{p} y_{0}}^{\prime} \mathrm{d} c_{\mathrm{g}_{0} x_{0}}, \\
& \mathrm{~d} \boldsymbol{p}_{\mathrm{e} 2}=c^{-2} \gamma^{-2} \boldsymbol{c}_{\mathrm{g}} \times \mathrm{d} \boldsymbol{m}_{\mathrm{p} 2}^{\prime}=\hat{z}_{0} c^{-2} \gamma^{-2} m_{\mathrm{p} y_{0}}^{\prime} \mathrm{d} c_{\mathrm{g} x_{0}}
\end{aligned}
$$

The partial derivative of the characteristic electric moment with respect to the velocity of the photon is (Equations (S47)-(S50))

$$
\left[\begin{array}{l}
\partial \boldsymbol{p}_{\mathrm{e}} / \partial c_{\mathrm{gx}_{0}} \\
\partial \boldsymbol{p}_{\mathrm{e}} / \partial c_{\mathrm{g} y_{0}} \\
\partial \boldsymbol{p}_{\mathrm{e}} / \partial c_{\mathrm{gz}_{0}}
\end{array}\right]=c^{-2}\left[\begin{array}{c}
\gamma^{-2}\left(\hat{z}_{0} m_{\mathrm{p} y_{0}}^{\prime}-\hat{y}_{0} m_{\mathrm{p} z_{0}}^{\prime}\right) \\
\left(3 \gamma^{-2}-2\right)\left(\hat{x}_{0} m_{\mathrm{p}_{0}}^{\prime}-\hat{z}_{0} m_{\mathrm{p} x_{0}}^{\prime}\right) \\
\gamma^{-2}\left(\hat{y}_{0} m_{\mathrm{px}_{0}}^{\prime}-\hat{x}_{0} m_{\mathrm{p} y_{0}}^{\prime}\right)
\end{array}\right]
$$

The total derivative of the characteristic electric moment is (Equation (S40), Equation (S51))

$$
\frac{\partial \boldsymbol{p}_{\mathrm{e}}}{\partial t}=\frac{\gamma^{-4} \partial B_{z} / \partial x}{h v_{\mathrm{s}}}\left[\begin{array}{c}
+\left(\hat{z}_{0} m_{\mathrm{py}_{0}}^{\prime}-\hat{y}_{0} m_{\mathrm{p}_{0}}^{\prime}\right) m_{\mathrm{p}_{0}}^{\prime} \cos \theta_{y} \\
+\gamma^{-1}\left(3 \gamma^{-2}-2\right)\left(\hat{x}_{0} m_{\mathrm{p}_{0}}^{\prime}-\hat{z}_{0} m_{\mathrm{p}_{0}}^{\prime}\right) m_{\mathrm{p}_{0}}^{\prime} \sin \theta_{y} \\
-\left(\hat{y}_{0} m_{\mathrm{px}_{0}}^{\prime}-\hat{x}_{0} m_{\mathrm{py}_{0}}^{\prime}\right)\left(m_{\mathrm{px}_{0}}^{\prime} \cos \theta_{y}+\gamma^{-1} m_{\mathrm{py}_{0}}^{\prime} \sin \theta_{y}\right)
\end{array}\right]
$$

In the state of thermal equilibrium the orientation of photon's proper magnetic moment is random, and the average productions of the components of photon's proper magnetic moment are

$$
\overline{m_{\mathrm{p} i}^{\prime} m_{\mathrm{p} j}^{\prime}}= \begin{cases}m_{\mathrm{p}}^{\prime 2} / 3 & i=j \\ 0 & i \neq j\end{cases}
$$

Then the average derivative of the characteristic electric moment with respect to time is

$$
\frac{\overline{\partial \boldsymbol{p}_{\mathrm{e}}}}{\partial t}=\frac{\gamma^{-4} \partial B_{z} / \partial x}{h v_{\mathrm{s}}} \frac{m_{\mathrm{p}}^{\prime 2}}{3}\left(\hat{x}_{0} \gamma^{-1}\left(3 \gamma^{-2}-1\right) \sin \theta_{y}-\hat{y}_{0} 2 \cos \theta_{y}\right)
$$

In the state of thermal equilibrium the directions distribution of the moving photons are random too, to transform the equation into the $(x, y, z)$ coordinate and to average it in spherical coordinates, then the average derivative of the characteristic electric moment of the photons with the same frequency is obtained.

$$
\frac{\overline{\partial \boldsymbol{p}_{\mathrm{e}}}}{\partial t}=-\hat{y} \frac{\gamma^{-4} \partial B_{z} / \partial x}{h v_{\mathrm{s}}} \frac{2 m_{\mathrm{p}}^{\prime 2}}{9}\left(1-\gamma^{-1}+3 \gamma^{-3}\right)
$$

\section{Method S7: The Curl of Electric Field Interacts with the Electric Moment of a Photon}

A moving photon has two transverse components of the characteristic electric moments (Figure S7, Figure S11, Equation (S45), Equation (S46))

$$
p_{\mathrm{ex}_{0}}=c^{-2} c_{\mathrm{g}} \gamma^{-2} m_{\mathrm{p} z_{0}}^{\prime}, \quad p_{\mathrm{ez}_{0}}=-c^{-2} c_{\mathrm{g}} \gamma^{-2} m_{\mathrm{p} x_{0}}^{\prime}
$$

Since the longitudinal component is zero and the transverse interaction is enlarged by one factor of relativity that is just like the situation of moving electron, so that the interacting electric moment of a moving photon is a tensor of second 
order,

$$
p_{\mathrm{e}_{0} j_{0}}=\left[\begin{array}{ccc}
p_{\mathrm{e}_{0} x_{0}} & p_{\mathrm{ex}_{0} y_{0}} & p_{\mathrm{ex}_{0} z_{0}} \\
0 & 0 & 0 \\
p_{\mathrm{ez}_{0} x_{0}} & p_{\mathrm{ez}_{0} y_{0}} & p_{\mathrm{ez}_{0} z_{0}}
\end{array}\right]=\left[\begin{array}{ccc}
\gamma p_{\mathrm{ex}_{0}} & p_{\mathrm{ex}_{0}} & \gamma p_{\mathrm{ex}_{0}} \\
0 & 0 & 0 \\
\gamma p_{\mathrm{ez}_{0}} & p_{\mathrm{ez}_{0}} & \gamma p_{\mathrm{ez}_{0}}
\end{array}\right]
$$

The first part of the proper force acted on the photon is the cross production of the electric moment and the curl of electric field and can be written in a clear form of determinant.

$$
f_{E 1}^{\prime}=\left|\begin{array}{ccc}
\hat{x}_{0} \gamma & \hat{y}_{0} & \hat{z}_{0} \gamma \\
p_{\mathrm{ex}_{0}} & 0 & p_{\mathrm{ez}_{0}} \\
\operatorname{curl}_{x_{0}} \boldsymbol{E} & \operatorname{curl}_{y_{0}} \boldsymbol{E} & \operatorname{curl}_{z_{0}} \boldsymbol{E}
\end{array}\right|
$$

The visual force acted on the photon by the curl of electric field is

$$
f_{E 1}=\left|\begin{array}{ccc}
\hat{x}_{0} & \hat{y}_{0} \gamma^{-2} & \hat{z}_{0} \\
p_{\mathrm{ex}_{0}} & 0 & p_{\mathrm{ez}_{0}} \\
\operatorname{curl}_{x_{0}} \boldsymbol{E} & \operatorname{curl}_{y_{0}} \boldsymbol{E} & \operatorname{curl}_{z_{0}} \boldsymbol{E}
\end{array}\right|
$$

and it can be calculated (Equation (S23)),

$$
f_{E 1}=c^{-2} c_{\mathrm{g}} \gamma^{-2} \frac{\partial E_{y}}{\partial x}\left|\begin{array}{ccc}
\hat{x}_{0} & \hat{y}_{0} \gamma^{-2} & \hat{z}_{0} \\
m_{\mathrm{p} z_{0}}^{\prime} & 0 & -m_{\mathrm{p} x_{0}}^{\prime} \\
\sin \phi_{y} \cos \theta_{y} & \sin \phi_{y} \sin \theta_{y} & \cos \phi_{y}
\end{array}\right|
$$

To transform the visual electric field into the characteristic magnetic field in proper system (Equation (S26)), then the interaction between the longitudinal magnetic moment and the curl of magnetic field in proper system is (Equation (S38))

$$
f_{E 2}^{\prime}=\left|\begin{array}{ccc}
\hat{x}_{0} \gamma & \hat{y}_{0} & \hat{z}_{0} \gamma \\
0 & m_{\mathrm{py}}^{\prime} & 0 \\
\operatorname{curl}_{x_{0}}^{\prime} \boldsymbol{B}_{\mathrm{c}, \boldsymbol{E}}^{\prime} & \operatorname{curl}_{y_{0}}^{\prime} \boldsymbol{B}_{\mathrm{c}, \boldsymbol{E}}^{\prime} & \operatorname{curl}_{z_{0}}^{\prime} \boldsymbol{B}_{\mathrm{c}, \boldsymbol{E}}^{\prime}
\end{array}\right|
$$

and it can be calculated (Equation (S27)),

$$
f_{E 2}^{\prime}=\hat{z}_{0} \gamma^{-1} c^{-2} c_{\mathrm{g}} m_{\mathrm{p} y_{0}}^{\prime} \cos \phi_{y} \sin ^{2} \theta_{y} \partial E_{y} / \partial x
$$

The second part of the visual force acted on the photon by the electric field is

$$
f_{E 2}=\hat{z}_{0} \gamma^{-2} c^{-2} c_{\mathrm{g}} m_{\mathrm{py}}^{\prime} \cos \phi_{y} \sin ^{2} \theta_{y} \partial E_{y} / \partial x
$$

The total visual force acted on the photon by the electric field is (Equation (S60), Equation (S63))

$$
f_{E}=c^{-2} c_{\mathrm{g}} \gamma^{-2} \frac{\partial E_{y}}{\partial x}\left(\begin{array}{c}
\hat{x}_{0} m_{\mathrm{p} x_{0}}^{\prime} \sin \phi_{y} \sin \theta_{y} \\
-\hat{y}_{0} \gamma^{-2}\left(m_{\mathrm{p} x_{0}}^{\prime} \sin \phi_{y} \cos \theta_{y}+m_{\mathrm{p} z_{0}}^{\prime} \cos \phi_{y}\right) \\
\hat{z}_{0}\left(m_{\mathrm{p} z_{0}}^{\prime} \sin \phi_{y} \sin \theta_{y}+m_{\mathrm{p} y_{0}}^{\prime} \cos \phi_{y} \sin ^{2} \theta_{y}\right)
\end{array}\right)
$$


Then the visual acceleration of the photon acted by the electric field is

$$
\left[\begin{array}{c}
\partial c_{\mathrm{g}_{0}} / \partial t \\
\partial c_{\mathrm{g}_{0}} / \partial t \\
\partial c_{\mathrm{gz}_{0}} / \partial t
\end{array}\right]_{E}=\frac{\gamma^{-3} c_{\mathrm{g}}}{h v_{\mathrm{s}}} \frac{\partial E_{y}}{\partial x}\left[\begin{array}{c}
m_{\mathrm{p} x_{0}}^{\prime} \sin \phi_{y} \sin \theta_{y} \\
-\gamma^{-2} m_{\mathrm{p} z_{0}}^{\prime} \cos \phi_{y}-\gamma^{-2} m_{\mathrm{p} x_{0}}^{\prime} \sin \phi_{y} \cos \theta_{y} \\
m_{\mathrm{p} z_{0}}^{\prime} \sin \phi_{y} \sin \theta_{y}+m_{\mathrm{p} y_{0}}^{\prime} \cos \phi_{y} \sin ^{2} \theta_{y}
\end{array}\right]
$$

Method S8: The Derivative of the Characteristic Magnetic Moment of the Photon

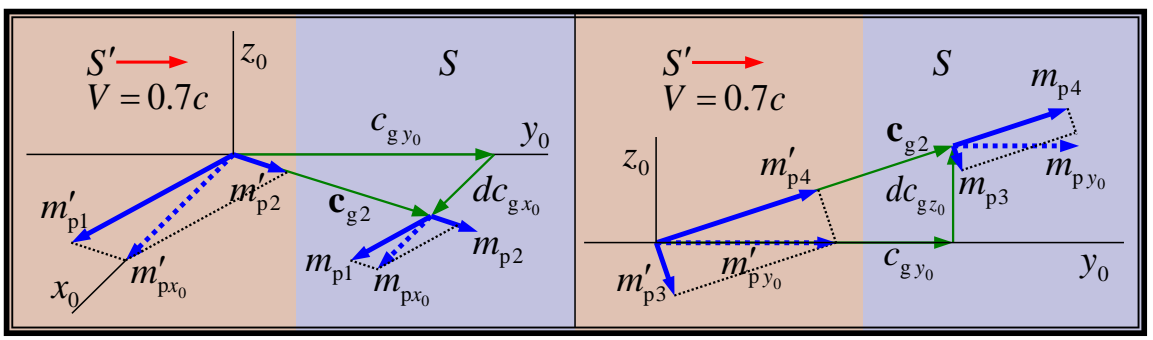

Figure S14. The change of the characteristic magnetic moment.

The characteristic magnetic moment in visual system is changed by the transverse acceleration of the photon. For the transverse magnetic moment on the left of the figure (Equation (19)),

$$
\begin{aligned}
& \mathrm{d} \boldsymbol{m}_{\mathrm{p}}\left(m_{\mathrm{p} x_{0}}^{\prime}\right)=m_{\mathrm{p} 1}+m_{\mathrm{p} 2}-m_{\mathrm{p} x_{0}} \\
& \mathrm{~d} \boldsymbol{m}_{\mathrm{p}}\left(m_{\mathrm{p} x_{0}}^{\prime}\right)=\gamma^{-2} m_{\mathrm{p} 1}^{\prime}+\gamma^{-1} m_{\mathrm{p} 2}^{\prime}-\gamma^{-2} m_{\mathrm{p} x_{0}}^{\prime} \\
& \mathrm{d} \boldsymbol{m}_{\mathrm{p}}\left(m_{\mathrm{p} x_{0}}^{\prime}\right)=\hat{y}_{0} c_{\mathrm{g}}^{-1}\left(\gamma^{-1}-\gamma^{-2}\right) m_{\mathrm{p} x_{0}}^{\prime} \mathrm{d} c_{\mathrm{g} x_{0}}
\end{aligned}
$$

and similarly

$$
\mathrm{d} \boldsymbol{m}_{\mathrm{p}}\left(m_{\mathrm{p} \mathrm{z}_{0}}^{\prime}\right)=\hat{y}_{0} c_{\mathrm{g}}^{-1}\left(\gamma^{-1}-\gamma^{-2}\right) m_{\mathrm{pz}_{0}}^{\prime} \mathrm{d} c_{\mathrm{gz}_{0}}
$$

For the longitudinal magnetic moment on the right of the figure (Equation (19)),

$$
\begin{aligned}
& \mathrm{d} \boldsymbol{m}_{\mathrm{p}}\left(m_{\mathrm{p} y_{0}}^{\prime}\right)=m_{\mathrm{p} 3}+m_{\mathrm{p} 4}-m_{\mathrm{p} y_{0}} \\
& \mathrm{~d} \boldsymbol{m}_{\mathrm{p}}\left(m_{\mathrm{p} y_{0}}^{\prime}\right)=\gamma^{-2} m_{\mathrm{p} 3}^{\prime}+\gamma^{-1} m_{\mathrm{p} 4}^{\prime}-\gamma^{-1} m_{\mathrm{p} y_{0}}^{\prime} \\
& \mathrm{d} \boldsymbol{m}_{\mathrm{p}}\left(m_{\mathrm{p} y_{0}}^{\prime}\right)=\hat{z}_{0} c_{\mathrm{g}}^{-1}\left(\gamma^{-1}-\gamma^{-2}\right) m_{\mathrm{p} y_{0}}^{\prime} \mathrm{d}_{\mathrm{g}_{0}}
\end{aligned}
$$

and similarly

$$
\mathrm{d} \boldsymbol{m}_{\mathrm{p}}\left(m_{\mathrm{py} 0}^{\prime}\right)=\hat{x}_{0} c_{\mathrm{g}}^{-1}\left(\gamma^{-1}-\gamma^{-2}\right) m_{\mathrm{py}}^{\prime} \mathrm{d} c_{\mathrm{g} x_{0}}
$$

The effect of longitudinal acceleration of the photon can be calculated directly.

Then the partial derivatives of the characteristic magnetic moment with respect to the velocity of the photon are (Equation (S66)-(S69))

$$
\begin{aligned}
& \partial \boldsymbol{m}_{\mathrm{p}} / \partial c_{\mathrm{gx}_{0}}=\hat{y}_{0} c_{\mathrm{g}}^{-1}\left(\gamma^{-1}-\gamma^{-2}\right) m_{\mathrm{p} x_{0}}^{\prime}+\hat{x}_{0} c_{\mathrm{g}}^{-1}\left(\gamma^{-1}-\gamma^{-2}\right) m_{\mathrm{p} y_{0}}^{\prime} \\
& \partial \boldsymbol{m}_{\mathrm{p}} / \partial c_{\mathrm{g}_{0}}=-\hat{x}_{0} 2 c_{\mathrm{g}} c^{-2} m_{\mathrm{p} x_{0}}^{\prime}-\hat{y}_{0} \gamma c_{\mathrm{g}} c^{-2} m_{\mathrm{p} y_{0}}^{\prime}-\hat{z}_{0} 2 c_{\mathrm{g}} c^{-2} m_{\mathrm{p} z_{0}}^{\prime} \\
& \partial \boldsymbol{m}_{\mathrm{p}} / \partial c_{\mathrm{gz}_{0}}=\hat{z}_{0} c_{\mathrm{g}}^{-1}\left(\gamma^{-1}-\gamma^{-2}\right) m_{\mathrm{py}_{0}}^{\prime}+\hat{y}_{0} c_{\mathrm{g}}^{-1}\left(\gamma^{-1}-\gamma^{-2}\right) m_{\mathrm{p} z_{0}}^{\prime}
\end{aligned}
$$


The derivative of the characteristic magnetic moment is (Equation (S65), Equation (S70))

$$
\frac{\partial \boldsymbol{m}_{\mathrm{p}}}{\partial t}=\frac{\gamma^{-3}}{h v_{\mathrm{s}}} \frac{\partial E_{y}}{\partial x}\left[\begin{array}{c}
\left(\gamma^{-1}-\gamma^{-2}\right)\left(\hat{y}_{0} m_{\mathrm{p} x_{0}}^{\prime}+\hat{x}_{0} m_{\mathrm{py}_{0}}^{\prime}\right) m_{\mathrm{p} x_{0}}^{\prime} \sin \phi_{y} \sin \theta_{y} \\
+\gamma^{-2} \beta^{2}\left(\hat{x}_{0} 2 m_{\mathrm{p} x_{0}}^{\prime}+\hat{y}_{0} \gamma m_{\mathrm{py}_{0}}^{\prime}+\hat{z}_{0} 2 m_{\mathrm{pz}_{0}}^{\prime}\right)\left(m_{\mathrm{p} 0_{0}}^{\prime} \cos \phi_{y}+m_{\mathrm{p} x_{0}}^{\prime} \sin \phi_{y} \cos \theta_{y}\right) \\
+\left(\gamma^{-1}-\gamma^{-2}\right)\left(\hat{z}_{0} m_{\mathrm{py}_{0}}^{\prime}+\hat{y}_{0} m_{\mathrm{pz}_{0}}^{\prime}\right)\left(m_{\mathrm{p} 0_{0}}^{\prime} \sin \phi_{y} \sin \theta_{y}+m_{\mathrm{py} 0}^{\prime} \cos \phi_{y} \sin ^{2} \theta_{y}\right)
\end{array}\right]
$$

In the state of thermal equilibrium the orientation of photon's proper magnetic moment is random, and then the average derivative is (Equation (S53))

$$
\frac{\overline{\partial \boldsymbol{m}_{\mathrm{p}}}}{\partial t}=\frac{\gamma^{-3}}{h v_{\mathrm{s}}} \frac{\partial E_{y}}{\partial x} \frac{m_{\mathrm{p}}^{\prime 2}}{3}\left[\begin{array}{c}
\left(\gamma^{-1}-\gamma^{-2}\right) \hat{y}_{0} \sin \phi_{y} \sin \theta_{y} \\
2 \gamma^{-2}\left(1-\gamma^{-2}\right)\left(\hat{x}_{0} \sin \phi_{y} \cos \theta_{y}+\hat{z}_{0} \cos \phi_{y}\right) \\
\left(\gamma^{-1}-\gamma^{-2}\right)\left(\hat{z}_{0} \cos \phi_{y} \sin ^{2} \theta_{y}+\hat{y}_{0} \sin \phi_{y} \sin \theta_{y}\right)
\end{array}\right]
$$

In the state of thermal equilibrium the directions distribution of the moving photons are random too, to transform the equation into the $(x, y, z)$ coordinate and to average it in spherical coordinates, then the average derivative of the characteristic magnetic moment of the photons with the same frequency is obtained.

$$
\frac{\overline{\partial \boldsymbol{m}_{\mathrm{p}}}}{\partial t}=\hat{z} \frac{\gamma^{-4} m_{\mathrm{p}}^{\prime 2}}{9 h v_{\mathrm{s}}} \frac{\partial E_{y}}{\partial x}\left(3+\gamma^{-1}-4 \gamma^{-3}\right)
$$

